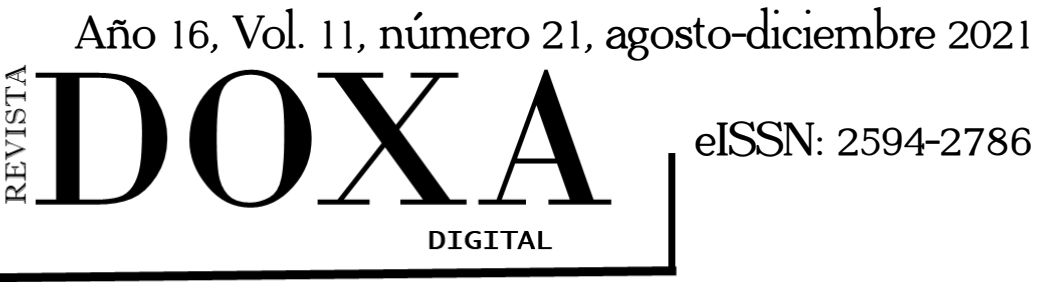

Sección: Humanidades

Recibido: Mayo 2021

Aceptado: Julio 2021

DOI: 10.52191/rdojs.2021.205

Pág.: 127-141

\title{
La permanencia académica: Un análisis desde la perspectiva estudiantil en el nivel licenciatura del área de Historia
}

\section{Academic tenure: An analysis from the student perspective at the undergraduate level in history majors}

\author{
Julio Cesar Gómez Gándara *
}

\section{RESUMEN}

El objetivo principal del presente trabajo es presentar los resultados obtenidos de una fase de un estudio exploratorio que forma parte de una investigación más amplia, y contribuyo al desarrollo de la problematización. Los resultados de dicho acercamiento exploratorio esbozan aquellos elementos que los alumnos consideraron como esenciales para su continuidad y permanencia al interior de la institución.

PAlabRAS ClaVe: Alumnos, Estudio Exploratorio, Permanencia académica, Universidad.

\section{ABSTRACT}

The main objective of this research is to present the results obtained from a phase of an exploratory study that is part of a broader research and contributed to the development of the problem. The results of this exploratory approach build the elements which the students considered essential for their continuity and permanence within the institution.

KEY WORDS: Students, Exploratory Study, Academic Permanence, University .

* Maestro en Educación y doctorante del programa de Educación por la Universidad Pedagógica Nacional del Estado de Chihuahua. Actualmente docente en la Facultad de Filosofia y Letras de la Universidad Autónoma de Chihuahua. Contacto: bhcampos13@gmail.com 
Año 16, Vol. 11, número 21, agosto-diciembre

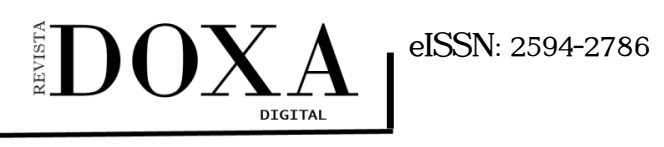

Sección: Humanidades

DOI: $10.52191 /$ rdojs.2021.205

Pág.: 127-141
Julio Cesar Gómez Gándara

La permanencia académica....

\section{Introducción.}

En las Instituciones Educativas el quehacer profesional, ético, moral y social que norma y regula las acciones en torno a la constitución de las mismas, idealmente podría proceder de los alumnos. Los denominados estudiantes son el motor generador y alimentador del proceso educativo, convirtiéndoles en uno de los principales actores de la educación. En su ingreso al nivel Superior, comienzan a generar información de índole académica, particular y profesional; esto con los resultados de las pruebas en los exámenes de selección, datos socioeconómicos, capacitaciones, preparación complementaria su formación, calificaciones, prácticas y servicios profesionales, entre algunas otras. Este tipo de información que es resultado de las actividades y acciones de los propios alumnos, no siempre es clara en la expresión de sus números y por tanto la falta de análisis o atención de los mismos desemboca en situaciones como la carencia de actualización de planes y programas así como de estrategias que favorezcan a los alumnos dentro de las Instituciones educativas.

El presente trabajo, se apoya en una realidad desde la subjetividad de los estudiantes, a partir de un acercamiento exploratorio, de aquello que reconocen, que contribuye a su permanencia dentro de su programa académico. Es por lo anterior, que el trabajo se centra en analizar, las características que presenta un grupo determinado en su integración y relación académica, analizando cómo estas favorecen la permanencia. Lo anterior es abordado dentro del contexto de la Licenciatura en Historia en la Facultad de Filosofía y Letras de la Universidad Autónoma de Chihuahua.

\section{Permanencia académica.}

Las situaciones que afrontan los alumnos universitarios, que muchas veces desembocan en el abandono, podrían ser la motivación central de las instituciones para focalizarse en la permanencia de los estudiantes y con ello hacer indispensable la comprensión de la dinámica del período escolar y diseñar estrategias, lo cual implica adaptarse a las necesidades académicas así como formativas del alumnado. El abordaje y la permanencia estudiantil dentro de la educación superior, contribuye a garantizar un programa académico encaminado a cobrar relevancia en el entorno universitario, mientras a la par persigue un crecimiento sólido, inclusivo y sostenible en una comunidad global.

Para definir la permanencia académica, Gairín (2015) la constituye como el conjunto de acciones que contribuyen a la consolidación del alumno en el programa educativo y en el proceso de continuación que lo lleva a concluir el plan de estudios. Leyva (2014) la orienta hacia los modos o 
Sección: Humanidades

DOI: $10.52191 /$ rdojs.2021.205

Pág.: 127-141
Julio Cesar Gómez Gándara

La permanencia académica....

estilos de actuación cotidianos, individuales y colectivos, propios del centro educacional, proceso que implica una adaptación al cambio constante, además de un aporte participativo en el sistema simbólico comunitario. Carballo (2017) utiliza el término persistencia que tiene el alumno en un programa educativo, hasta lograr su título o grado académico. La permanencia no tiene unicidad semántica ante este escenario, el término primario abduce a un proceso simple del estudiante que inicia una carrera hasta concluirla, después se ve enriquecido con plantear un momento inicial y otro de continuidad hasta que la inserción cultural dentro de la institución con el aprendizaje simbólico ocurre.

Los autores Leyva (2014), Gairín (2015) así como Carballo (2017), caracterizan en un común denominador la permanencia de los alumnos, el cual considera que los estudios son cursados de una manera regular, que no fueron abandonados o pausados, que no han cambiado de carrera y/o unidad académica dentro de su institución u otra. Con ello, cuando se habla de un alumno regular, se entenderá una definición administrativa que consiste en aquella situación del estudiante que cursa sus estudios universitarios bajo dichos requerimientos.

Algunos autores han relacionado permanencia con retención escolar como Fonseca y García (2016), al concebir que ambos términos significan que el estudiante persiste en el programa educativo hasta que egresa. No obstante, la retención alude a algo o alguien que ejecuta acciones externas al individuo para retenerlo y la permanencia plantea la persistencia en términos de modificar la realidad para permanecer en el programa pese a un contexto adverso. Como lo destacan Torres (2010) y Viale (2014), la permanencia en los estudios por parte de los alumnos es consecuencia de la interacción entre el alumno como individuo y la institución como una organización, destacando la importancia de esta interacción a partir del significado que cada estudiante le atribuye a su relación con las dimensiones formales e informales de cada institución.

Uno de los factores relacionados con la permanencia son las trayectorias escolares. Gartner y Gallego (2013) mencionan que estas implican contemplar el grado de conocimientos que posee un individuo de un determinado nivel que emplea como resultado de ser estudiante y son reconocidos por los otros. El seguimiento de las denominadas trayectorias escolares, como lo enmarcan Cain y Ramírez (1997), hace referencia al nivel de rendimiento, aprovechamiento así como de los logros escolares; contemplando los éxitos y los fracasos de los alumnos así como de los actores implicados en ellos. Por su parte, este rendimiento se ve asociado a otro tipo de factores como motivación, metas objetivos y logros trazados por el estudiante que derivan en que el alumno continúe o 
Año 16, Vol. 11, número 21, agosto-diciembre

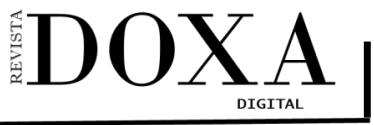

Sección: Humanidades

DOI: $10.52191 /$ rdojs.2021.205

Pág.: 127-141
Julio Cesar Gómez Gándara

La permanencia académica....

no su preparación académica.

La permanencia académica se ve altamente influenciada por el nivel mostrado en la integración académica de cada estudiante. Donoso y Schiefelbein (2007) exponen que el nivel de integración es ponderada por los propios alumnos en base a la cantidad de compromiso requerido, reflejado en las recompensas obtenidas que son valoradas sobre el tiempo invertido en este proceso. En complemento con esto, también es señalado por los autores Gónzalez, Sibelli y Montalvo (2007), que el modelo de integración académica tiene dos componentes: los resultados o calificaciones obtenidas en el ciclo de estudio; y el segundo la integración social que se mide por el número de participaciones o actividades no obligatorias con sus compañeros y profesores. Por tal motivo cada alumno en lo singular, tendrá diversos factores asociados a su decisión y situación escolar que le determine para poder seguir adelante con sus estudios dentro del nivel de licenciatura.

El dar seguimiento a las trayectorias escolares desde la integración académica del alumno, permitirá a la institución tener un panorama claro para identificar las posibles estrategias de acción en relación a sus estudiantes. El visualizar a los estudiantes en su comportamiento dentro de las instituciones, permitirá priorizar los niveles de atención, estrategias y asertividad para contribuir a la permanencia académica.

\section{Acercamiento al problema.}

El sistema de Educación Superior en México es amplio y ha experimentado un crecimiento acelerado y sostenido. La SEP (2019) estima que en el año 2019 se contaba con cerca de 4.4 millones de estudiantes; 3.8 millones de estudiantes en programas presenciales y 0.6 millones en programas a distancia o en línea, presentes en más de 7000 escuelas y casi 38000 programas de educación a nivel superior. La OECD (2020) destaca que el sistema de educación superior mexicano, que alcanza los 36 millones de estudiantes, en la actualidad prevé que solo el $56,3 \%$ de los matriculados termine este nivel de estudios.

Dentro de los estudios realizados a las preferencias académicas de los jóvenes, la OECD (2017) subraya que las elecciones de estudio más recurrentes por los alumnos son Derecho y Administración, con un 35.1\% de matriculados; seguidos de las Ingenierías con el 24.4\%; le prosiguen los programas de Salud con un 10.1\%; las Ciencias Naturales presentan un $3.1 \%$ de los matriculados mientras que Artes y Humanidades atiende al 1.9\% al igual que el área de Información y Comunicación. 
Año 16, Vol. 11, número 21, agosto-diciembre

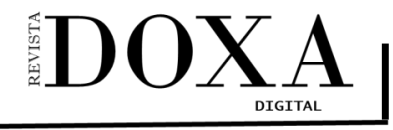

Sección: Humanidades

DOI: $10.52191 /$ rdojs.2021.205

Pág.: 127-141
Julio Cesar Gómez Gándara

La permanencia académica....

Velázquez y González (2017) subrayan que bajo este enfoque interaccionista, cuando el alumno decide abandonar se debe a que los beneficios percibidos para permanecer en la institución son inferiores a los costos personales derivados del quehacer universitario, o bien las interacciones sociales y académicas no resultaron suficientemente satisfactorias.

Con base en la observación referida en el estudio de las Humanidades como panorama de recurrencia menor en la elección de estudio de los universitarios, la Facultad de Filosofía y Letras de la Universidad Autónoma de Chihuahua afronta una problemática dentro de su programa de formación de Licenciatura en Historia. En esta licenciatura se detecta un decrecimiento en la cantidad de alumnos que permanecen y continúan una vez que ingresan al plan de estudios; incluso es posible señalar que en más de una ocasión se han quedado sin egresar generaciones de los alumnos que forman parte del programa académico en su modalidad presencial. La licenciatura atraviesa un fenómeno de baja continuidad escolar, asociado a la no permanencia que se tiene por parte de los alumnos que ingresan y que no continúan con su formación profesional dentro de esta opción de estudio.

Basándose en lo anteriormente registrado dentro de la licenciatura en mención, es que se decide hacer una ejemplificación del comportamiento de continuidad escolar de manera semestral en cuatro de los semestres recientes de la Licenciatura en Historia. Para conocer la situación se realizó un análisis de los alumnos formalmente inscritos desde enero del 2018 hasta agosto 2019; es decir cuatro generaciones. Para ello se considera la situación académica de cada estudiante que lo ubique en el semestre correspondiente acorde a sus materias acreditadas. Uno de los factores importantes para determinar el semestre en el que se encuentra el alumno, es si adeuda alguna materia, lo cual lo ubica en el semestre más bajo al que corresponda en la carrera.

El decremento de la permanencia mostrado en la Figura 1, indica mayores valores en los semestres sucesivos al quinto y especifica que más de la mitad de los alumnos no continua en el programa académico en esta modalidad. Se observa que del sexto al noveno semestre de la Licenciatura hay una población estudiantil del $20 \%$ en relación a la población ingresada al programa académico.

En el semestre enero - junio del 2018 la población inscrita en el programa académico de Historia presencial fue de 68 alumnos; sin embargo del sexto al noveno semestre la población académica era de seis alumnos, lo que representó solo un $8 \%$ de la totalidad de los inscritos dentro del programa en ese momento. En el sexto semestre no hay alumnos registrados que conformarán par- 
Año 16, Vol. 11, número 21, agosto-diciembre Sección: Humanidades

DOXA
DOI: $10.52191 /$ rdojs.2021.205

Pág.: 127-141
Julio Cesar Gómez Gándara

La permanencia académica....

te de esa generación. Para el siguiente semestre que data de agosto - diciembre del 2018 la cantidad de alumnos matriculados en esos semestres fue de ocho, lo que representó un $12 \%$ de la población estudiantil que en ese momento era de 63 alumnos. En el octavo semestre nuevamente no se contaba con alumnos matriculados.

Figura1. Comportamiento de la permanencia de cuatro generaciones de la Licenciatura en Historia presencial de la FFyL - UACH

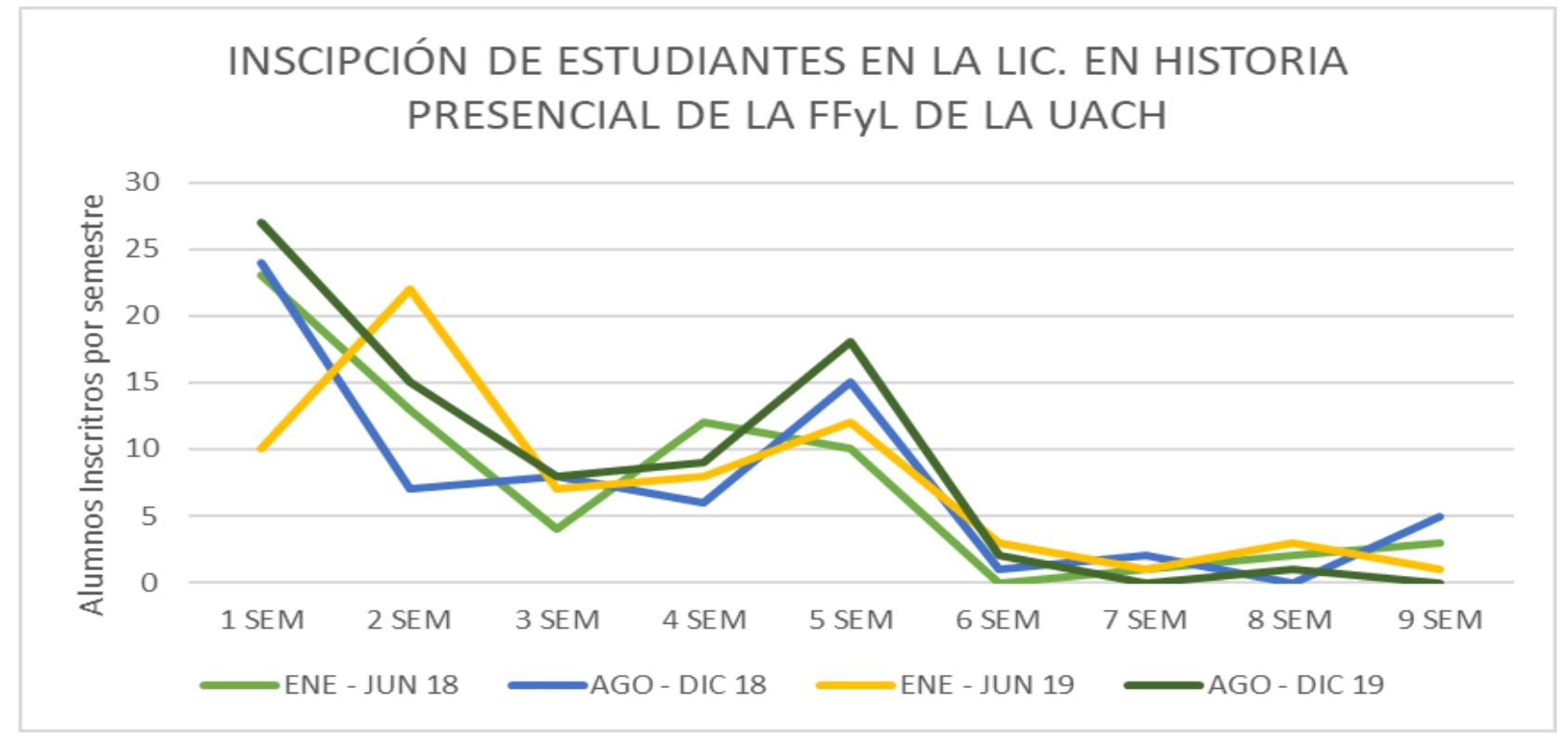

Fuente: Elaboración propia con base en datos proporcionados por la FFyL

Para inicios del primer semestre del año 2019 se contó con una matrícula de 66 alumnos, sin embargo la cantidad de alumnos de sexto a noveno semestre fue nuevamente de ocho alumnos, quienes representaron el $11 \%$ de la población. Para el semestre agosto - diciembre del 2019, la cantidad de alumnos inscritos en los semestres de sexto a noveno semestre fue de tres, quienes representan el $4 \%$ del total. Es importante señalar que en séptimo y noveno no hubo alumnos. La cantidad de alumnos que ingresaron al programa durante este ciclo tuvo un aumento en comparación al ingreso de otros semestres con una demanda de 29 aspirantes aceptados, 27 pagaron su inscripción y se sumaron a los 53 alumnos registrados en la Licenciatura de segundo a noveno semestre. Ello representó un ingreso del $33 \%$ de la totalidad de alumnos inscritos en el programa.

Aun cuando la deserción escolar es una problemática de la educación no solo de este programa académico, sino de todo el país; los estudios realizados en torno a los factores que inciden en la permanencia de los alumnos dentro de las instituciones universitarias son pocos. Este fenó- 
Sección: Humanidades

DOI: 10.52191/rdojs.2021.205

Pág.: 127-141
Julio Cesar Gómez Gándara

La permanencia académica....

meno no se ha indagado suficientemente, ya que se ha limitado únicamente a la compilación, conjunción, exposición y análisis de los datos estadísticos que cada institución produce de manera ordinaria sin buscar las causas reales que llevan a los estudiantes a tomar la decisión de permanecer y continuar. Los datos pueden ser sesgados debido a la poca seriedad que aportan los alumnos de las razones por las cuales se retiran o permanecen, información que puede no ser clara, confusa o abarca un ámbito muy general en las bases numéricas.

Como se menciona en las afirmaciones anteriores, el decremento en la cantidad de alumnos que continúan sus estudios de la Licenciatura en Historia es notorio, en comparación con la matrícula de las demás licenciaturas de la misma Unidad Académica. Para concebir el problema de investigación es necesario que holísticamente se estudien los diversos factores que de manera combinada o particular infieren en la determinación de los alumnos para continuar con su plan de estudios. En el presente estudio se entenderá por desertores a los alumnos que causan baja, abandonan, cambian de modalidad, de carrera o de unidad de estudios dentro de la UACH; y se entenderá por alumnos permanentes a aquellos que continúen de manera ininterrumpida sus estudios dentro del plan académico.

\section{El estudio exploratorio.}

En esta investigación se coloca al estudiante en un punto de análisis y reflexión centrado en su cotidianeidad escolar, dotándole de relevancia al proceso de interacción y relación que sostiene con su grupo. Los alumnos, como protagonistas del proceso de enseñanza - aprendizaje, son llevados al primer plano a través del presente trabajo para contemplar su perspectiva, su opinión y su relación cotidiana con la institución.

Con el propósito de conocer el panorama que visualiza población estudiantil en torno a sus aspiraciones e intenciones de permanecer dentro del programa académico, se realizó un estudio exploratorio a manera de diagnóstico. El objetivo de dicho instrumento tenía por intención, valorar la importancia e influencia que le otorgan estos alumnos a distintos factores en su entorno académico, profesional, social y personal. Partiendo de las respuestas arrojadas por la investigación (ver anexo 1) durante septiembre del 2019 a los alumnos de primer semestre de la Licenciatura en Historia presencial.

La población objetiva de este análisis será comprendida por los alumnos de nuevo ingreso en plan presencial de la Licenciatura en Historia de la FFyL, los cuales comprenden 27 matrículas acti- 
Sección: Humanidades

DOI: 10.52191/rdojs.2021.205

Pág.: 127-141
Julio Cesar Gómez Gándara

La permanencia académica....

vas en su primer semestre. Con el fin de estimar las causas que llevan a la permanencia de los alumnos dentro del programa académico de estudios señalado, con base en las comparativas del comportamiento atípico de la matricula que presenta la Licenciatura en Historia, es que se procede a caracterizar a los sujetos de estudio. De los 27 alumnos que ingresaron en su primer semestre, se tiene una distribución de 19 hombres y 8 mujeres con edades que van de los 17 años hasta los 66 años de edad.

El diseño del instrumento se apoya en el modelo de análisis propuesto por Christian Díaz (2008), en el cual se destaca la valoración de elementos preuniversitarios, institucionales, familiares, individuales y las expectativas laborales presentes en el alumno para considerar su permanencia. Señala que no existe una única variable que infiera en la decisión de los estudiantes, sino que es una combinación de "los factores externos, la motivación y las percepciones de la enseñanza que muestran estrechas relaciones en los estudiantes al tomar las decisiones de abandono o de permanencia." (2008: 74).

El instrumento creado para recoger la información por parte del grupo que conforma la población objetiva, se ve articulado por una escala tipo Likert. Dichas escalas "son instrumentos psicométricos donde el encuestado debe indicar su acuerdo o desacuerdo sobre una afirmación, ítem o reactivo, lo que se realiza a través de una escala ordenada y unidimensional." (Matas, 2018:39). Este tipo de escalas permiten tener mayor coherencia, accesibilidad y oportunidad de interpretación en los resultados arrojados dentro de los estudios. Con la información se creó una base de datos procesada de manera manual en el programa Excel del paquete computacional Office para equipos electrónicos.

\section{Análisis de los datos.}

De acuerdo a los datos obtenidos mediante el instrumento aplicado a los alumnos de nuevo ingreso de la Licenciatura en Historia presencial en el semestre 2019-B, de los 27 alumnos que representan el total de la población de nuevo ingreso al programa de estudios, todos manifestaron un estado civil de solteros. De los resultados arrojados, la mayoría de los estudiantes que conforman la población objetivo ronda entre los 17 y 20 años de edad, por lo que representó el $82 \%$. El $18 \%$ de los alumnos se comporta en edades entre los 23 y 66 años de edad. Los alumnos que cuentan con algún empleo representan el $15 \%$ del total, el resto expresó no estar laborando de manera profesional o informal a su ingreso. 
Año 16, Vol. 11, número 21, agosto-diciembre

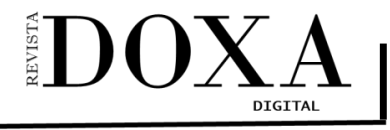

Sección: Humanidades

DOI: $10.52191 /$ rdojs.2021.205

Pág.: 127-141
Julio Cesar Gómez Gándara

La permanencia académica....

El $11 \%$ de los alumnos cuenta con algún tipo de estudio de nivel profesional. Las respuestas que expresaron en el instrumento fueron: Técnico en Mecatrónica, Diplomado en Literatura así como Licenciado en Filosofía. Las tres personas que manifestaron contar con estudios previos, dos de ellas los realizaron en áreas de estudio relacionadas con las Humanidades y afines al perfil de la Licenciatura en Historia. El resto del grupo representado en los otros 24 estudiantes, no cuenta con algún título profesional. Este resultado puede ser observable si se considera la edad de los alumnos que ingresan apenas rebasa los 18 años, por tanto la probabilidad de contar con algún título profesional es menor.

Las respuestas a la pregunta puntual que cuestionaba a los alumnos las razones que les motivaron para ingresar a la Licenciatura en Historia en su modalidad presencial, fueron agrupadas considerando su contenido. Se señala que las respuestas otorgadas en el instrumento se contestaron de manera individual, escrita y de extensión libre. Dichas enunciaciones se segmentaron en cinco áreas afines de respuesta: la investigación, la docencia, la gestión cultural, el medio social y segunda opción. La docencia tuvo un 37\%, mientras que la investigación como la gestión cultural tuvieron una preferencia del $22 \%$ cada una; lo que expresa el deseo de desempeñarse profesionalmente en alguna de estas tres áreas formativas en el perfil de egreso del programa. El medio social agrupó las respuestas en las cuales su ingreso al programa académico se influencio por algún factor externo o personal lo que representó el $11 \%$ y el ingreso por segunda opción de programa tuvo el $8 \%$.

Los alumnos que ingresaron buscando especializarse en alguna de las áreas formativas del programa académico, visualizan el área de investigación en la publicación de libros, trabajo en archivo y análisis histórico. La docencia en niveles de educación media superior y superior. La gestión cultural desde la difusión, preservaciones y extensionismo. Esta población representó el $81 \%$, lo que indica que más de la mitad conoce las áreas de oportunidad del programa académico al que ingresó y sabe en que desea especializarse.

Del total de los alumnos de nuevo ingreso, el $44 \%$ expreso que tiene un interés muy alto en estudiar y el $33 \%$ un interés alto; dándonos un $77 \%$ que denota una intención positiva en torno al programa académico. El 19\% manifestó un interés regular por continuar con su estudio y el $4 \%$ una intención muy baja por el programa. Ninguno señaló un interés bajo en su respuesta.

El interés mostrado por los alumnos de nuevo ingreso en culminar su programa académico 
Año 16, Vol. 11, número 21, agosto-diciembre

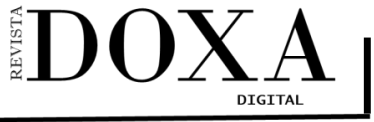

Sección: Humanidades

DOI: $10.52191 /$ rdojs.2021.205

Pág.: 127-141
Julio Cesar Gómez Gándara

La permanencia académica....

expuso que el $59 \%$ tiene un interés muy alto y el $22 \%$ un interés alto; lo que arroja que el $81 \%$ de la intención se ve canalizada en egresar. El 4\% enfatizó un interés bajo, mientras que el 15\% enunció un interés regular por culminar el programa. Ninguno señaló un interés muy bajo en su respuesta.

El nivel de influencia que le dieron los alumnos de nuevo ingreso a sus compañeros de clase con respecto a su permanencia en su mayoría es regular con un 41\% de la decisión. El 15\% considera que sus compañeros tendrán un nivel de influencia muy alto en su continuidad, mientras que el 18\% expresa un nivel alto; es decir, el 33\% de los estudiantes le otorga relevancia a la relación que se establezca en el salón de clases para continuar su paso por esta Licenciatura. El 11\% manifestó que sus compañeros jugarán un nivel de influencia muy bajo y el 15\% mencionó que sus compañeros tendrán un papel bajo en su permanencia.

Los alumnos de nuevo ingreso expresaron que el nivel de influencia que tendrán los profesores que imparten clase en la Licenciatura con respecto a su permanencia en el programa es importante. La opinión en relación a que el nivel de influencia será muy alto y alto comparte un $26 \%$ cada uno, lo que representa un 52\% de importancia entorno a la figura del profesor. El 44\% consideró que el nivel de influencia regular y solo el $4 \%$ manifestó que será de un nivel muy bajo. Ninguno señaló un nivel bajo en su respuesta.

El nivel de influencia con el que los alumnos ponderan a la familia en torno a su permanencia en la licenciatura es regular, ya que el 33\% manifestó estar de acuerdo en ello. El 26\% dijo darle una ponderación alta, mientras que el 19\% la colocaron en una escala muy alta. Fue el 15\% que opto por darle una valoración baja así como el 7\% una muy baja.

En relación al nivel de influencia que los amigos tendrán en su permanencia, apunta que el $30 \%$ considera que jugarán un papel bajo y el 26\% uno muy bajo; es decir que el $56 \%$ de las opiniones no ve que sus amistades tengan algún tipo de injerencia en su continuidad. El $26 \%$ le da un valor regular al papel de los amigos, mientras que el $7 \%$ y el $11 \%$, le brinda un nivel de importancia alto y muy alto respectivamente. Ninguno señaló un nivel regular en su respuesta. .

La influencia que representa conseguir un empleo para su permanencia en el programa, concentró un 30\% del grupo en un nivel de importancia alto. El 7\% consideró un nivel de importancia muy alto; lo que marca que el $37 \%$ del grupo considera importante el campo laboral en torno a la Licenciatura para continuar estudiando. El $41 \%$ señala como regular el nivel de influencia que esto representaría, mientras que en un valor bajo y muy bajo las posibilidades laborares en torno a la li- 
Sección: Humanidades

DOI: 10.52191/rdojs.2021.205

Pág.: 127-141
Julio Cesar Gómez Gándara

La permanencia académica....

cenciatura representaron un $11 \%$ cada una.

La influencia que la obtención de una beca para permanecer en sus estudios fue valorada en un nivel muy alto por el $26 \%$. El $33 \%$ de los alumnos denotó que el recibir algún apoyo o estimulo será de alto grado de influencia. Otro $33 \%$ dijo que el ser acreedor a una beca académica les resultaría en un nivel regular de importancia. En una ponderación de muy bajo, el $8 \%$ señalo ese nivel de influencia en su decisión. Ninguno señaló un nivel bajo en su respuesta.

La exploración que se hizo también incluyó comentarios y observaciones adicionales. En este punto del instrumento se busca que los alumnos expresen alguna observación, sugerencia o señalamiento puntual en torno a lo que esperan del programa académico. Las respuestas descritas fueron contestadas de manera individual, escrita, de extensión libre y agrupadas considerando sus características. Los resultados se agruparon en cuatro áreas de respuesta: Aquellos que decidieron no expresar algún comentario, quienes utilizaron el espacio para realizar sugerencias, quienes detallaron algunas observaciones y quienes decidieron aprovechar la oportunidad para hacer planteamientos personales.

Un $52 \%$ que representa decidió no utilizar el espacio. El $11 \%$ opto por realizar sugerencias para la coordinación de la licenciatura. El 7\% aprovecho el espacio para realizar observaciones de comportamiento tanto de alumnos como de profesores; mientras que un $30 \%$ decidió expresar muestras de aprobación, sentimientos y preferencias en relación al programa académico.

\section{Resultados.}

Del grupo de alumnos recién ingresados a la Licenciatura en Historia en modalidad presencial en la generación 2019 se identifica que el $82 \%$ del grupo tiene una edad entre 17 y 20 años, por lo que se advierte un grupo joven. El $81 \%$ de los alumnos tiene definida alguna meta que lo llevó a elegir la Licenciatura en Historia para prepararse laboralmente; es decir, tiene la intención de desempeñarse profesionalmente para ejercer en alguna de las áreas de oportunidad que ofrece el perfil de egreso de la Licenciatura en Historia. El $77 \%$ del grupo denota que tiene una intención positiva por estudiar, lo que conjunta las respuestas de la ponderación alta y muy alta en torno al programa académico.

En las preguntas en las que se buscaba evidenciar el nivel de influencia que juegan aspectos sociales y personales que competen al alumno en torno a su permanencia como lo son los compa- 
Sección: Humanidades

DOI: $10.52191 /$ rdojs.2021.205

Pág.: 127-141
Julio Cesar Gómez Gándara

La permanencia académica....

ñeros, la familia, los profesores, los amigos, las becas y las oportunidades laborales, se concluyó lo siguiente:

El $41 \%$ consideró una influencia regular a sus compañeros de clase en su decisión de permanecer. El 52\% consideró importante, al contestar como respuestas que la influencia por parte de sus profesores de clase en su decisión de permanecer será muy alta y alta. El 33\% consideró una influencia regular por parte de su familia en su decisión de permanecer. El 56\% consideró una influencia muy baja y baja en sus respuestas relacionadas al nivel de influencia que representan los amigos en su decisión de permanecer. El 41\% consideró una influencia regular el conseguir un empleo en su decisión de permanecer. El 33\% consideró un nivel de influencia muy alto el hecho de conseguir alguna beca en su decisión de permanecer.

Los alumnos que cuentan con algún empleo representa el 15\% del total, el resto expresó no estar laborando de manera profesional o informal a su ingreso. El 11\% de los alumnos cuenta con algún otro tipo de estudio de nivel profesional. El $81 \%$ del grupo tiene intenciones de egresar del programa académico. Por último, el $52 \%$ de los alumnos prefiero no utilizar el espacio de comentarios disponible al final del instrumento.

\section{Conclusiones.}

Este estudio exploratorio, como ya fue presentado al inicio del documento, forma parte de una investigación doctoral más amplia que se encuentra en desarrollo; sin embargo, esta indagación previa permitió hacer una aproximación, desde la perspectiva de los estudiantes a las situaciones que viven y que hacen posible su permanencia. Esta forma de abordar el tema de investigación es novedosa respecto de la forma de otras investigaciones en las que los autores establecen a priori los elementos a analizar. La perspectiva fue basarse en la información proveniente de los alumnos .Lo anterior es el motivo central de caracterizar como objeto del estudio a los alumnos que conforman la generación que ingresaron en el semestre Agosto - Diciembre en el año 2019 a la Licenciatura en Historia y a partir de su perspectiva generar análisis y reflexiones.

Los costos personales, recompensas, habilidades, motivación y la pertenencia e identificación que presente el alumno en su paso por la institución, podrían ser elementos a favor de la continuidad educativa. La perspectiva del alumno cobra relevancia debido a la poca profundidad en los estudios previos y escasas publicaciones de los mismos, basados desde la visión del alumno; por 
Sección: Humanidades

DOI: 10.52191/rdojs.2021.205

Pág.: 127-141
Julio Cesar Gómez Gándara

La permanencia académica....

lo cual se da pie a futuras indagaciones de rigor académico mediante un acompañamiento exploratorio del tema, que rescate y exponga los elementos académicos e institucionales desde lo expresado por los alumnos que contribuyen a su permanencia en semestres posteriores dentro de la Licenciatura.

La conveniencia de la elaboración de este tipo de investigación, yace en el compromiso de la Universidad como Institución de Educación Pública Superior, mismo que le orienta a ofertar programas de calidad, emplear la universalidad de la educación al igual que coadyuvar con el desarrollo sustentable, social y económico de su entidad. La relevancia metodológica del estudio orienta a visualizar modelos que estudien la vida natural y cotidiana de los alumnos en su entorno cercano, no condicionando a momentos específicos; como resultado se aportará una construcción metodológica para abordar la realidad de este tipo de situaciones particulares, apoyándose en estudios y modelos teóricos que otros investigadores han aportado.

Dentro de la relevancia social que presenta la investigación, en primera instancia se centra en brindar un acercamiento a las interacciones que mantienen los estudiantes como miembros activos de la Unidad Académica, como agentes protagónicos de las relaciones grupales e institucionales y como estos se verán reflejados en el ambiente laboral y profesional. Dicho proyecto de investigación puede abonar a los parámetros descritos dentro del Plan de Desarrollo Universitario de la Institución, que puntualmente contiene el marco de los valores y principios de una universidad socialmente responsable. Trabajar a partir de los resultados arrojados de instrumentos evaluatorios diseñados por las instituciones, que rescaten los posicionamientos de sus alumnos, puede dar como resultado un cumulo de estrategias, recursos y elementos; con ello se busca generar el desarrollo de estrategias y planes de mejora dentro de la institución.

La utilidad metodológica que tendrá el documento servirá como referente en futuros estudios que se lleven a cabo no solo dentro del área de Educación, sino también en el área de las Humanidades. Este es aunado al poco conocimiento que se tiene de este orden de estudios, los cuales son limitados y poco compartidos en la realidad de investigación, entendiendo que la información y los datos contenidos son de carácter confidencial bajo un orden institucional; por ello los resultados, propuestas o planes de acción emprendidos por las instituciones de educación que llegan a realizar estudios de esta índole son raras vez compartidos y expuestos a la comunidad. 
Año 16, Vol. 11, número 21, agosto-diciembre

\section{DOXA}

Sección: Humanidades

DOI: $10.52191 /$ rdojs.2021.205

Pág.: 127-141
Julio Cesar Gómez Gándara

La permanencia académica....

\section{Referencias.}

Cain, R. y Ramírez, C. (1997). Trayectoria escolar: La eficiencia terminal en la Universidad Veracruzana. ANUIES, Ed. Revista de la Educación Superior, XXVI(102), pp. 1-11. Recuperado el 11 de Abril de 2021, de http:// publicaciones.anuies.mx/pdfs/revista/Revista102_S2A2ES.pdf

Carballo, O. (2017). Factores que inciden en la permanencia académica de estudiantes a distancia en el nivel superior. Caso de estudio: Licenciatura en Estadística y Sistemas de Información. Revista Iberoamericana de Producción Académica y Gestión Educativa , IV(8). Recuperado el 05 de Abril de 2021, de http://pag.org.mx/index.php/PAG/ article/view/694/925

Donoso, S. y Schiefelbein, E. (2007). Análisis de los modelos explicativos de retención de estudiantes en la Universidad:Una visión desde la dsigualdad social. Estudios Pedagógicos XXXIII, pp. 7-27.

Díaz, C. (2008). Modelo concpetual para la desercion estudiantil Universitaria Chilena. Estudios Pedagógicos, XXXIV(II), pp. 65-86. Recuperado el 23 de Abril de 2021, de https://scielo.conicyt.cl/scielo.php? script=sci_abstract\&pid=S0718-07052008000200004\&lng=es\&nrm=iso

Gairín, J. (2015). Los sistemas de acceso, normativa, permanencia y estrategias de tutoría y retención de estudiantes en educación superior. Madrid, España: Wolters Kluwer. Recuperado el 29 de Abril de 2020, de https:// ddd.uab.cat/record/132953

Gartner, M. y Gallego, C. (2013). ¿Es la permanencia estudiantil un asunto de calidad educativa? III Conferencia Latinoamericana sobre el abandono en la Educación Superior. Estado de México, México. Recuperado el 11 de Abril de 2021, de http://clabes-alfaguia.org/clabes-2013/ponencias/LT_3/ponencia_completa_45.pdf

Gónzalez, C.; Sibelli, A. y Montalvo, D. (2007). Integración Académica y Social de Individuos . Enseñanza e Investigación Psicologíca, XII(2), pp. 237-257. Recuperado el 4 de Junio de 2020, de https://www.redalyc.org/articulo.oa? idp $=1 \& i d=29212202 \&$ cid $=85373$

Leyva, J. (2014). La permanencia escolar en las redes educacionales desde comunidades periféricas. Panorama, XV (8), 48-57.

Matas, A. (2018). Diseño del formato de escalas tipo Likert: un estado de la cuestión. Revista Electronica de Investigación Educativa , 20(1), pp. 38-47. Recuperado el 23 de Abril del 2021 de : dhttps://doi.org/10.24320/ redie.2018.20.1.1347

OECD. (2017). Panorama de la Educación . México: Nota País. Recuperado el 22 de Abril de 2021, de https:// www.oecd.org/education/skills-beyond-school/EAG2017CN-Mexico-Spanish.pdf

OECD. (2020). Education at a Glance 2020: OECD Indicators. OECD Publishing, Paris. Recuperado el 11 de Abril de 2021, de https://www.oecd-ilibrary.org/education/education-at-a-glance-2018_eag-2018-en

SEP. (2019). Sistema Educativo de los Estados Unidos Mexicanos: Principales Cifras 2018-2019 . México.: Secretaria de Educación Pública. Recuperado el 15 de Mayo de 2020, de https://www.planeacion.sep.gob.mx/ estadisticaeindicadores.aspx

Torres, L. (2010). Estado del arte de la retención de estudiantes de la educación superior. Tesis, Pontificia Universidad Javeriana, Facultad de Educación , Bogotá, D.C. Recuperado el 17 de Octubre de 2020, de https:// es.scribd.com/document/246325995/Estado-Del-Arte-de-La-Retencion-de-Estudiantes 
Año 16, Vol. 11, número 21, agosto-diciembre Sección: Humanidades

DOXA

Pág.: 127-141
DOI: $10.52191 /$ rdojs.2021.205

Julio Cesar Gómez Gándara

Viale, H. (2014). Una aproximación teórica a la deserción estudiantil universitaria . Revista Digital de Investigación en

Docencia Universitaria, VIII(1), pp. 59-75.

Velázquez, Y., \& Gónzalez, M. (2017). Factores asociados a la permanencia de estudiantes universitarios: caso UAMM-

UAT. Revista de la Educación Superior, XLVI(184), pp. 117-138.

\section{Anexo 1.}

Anexo 1. Instrumento utilizado para recabar la información pertinente de los alumnos de la Licenciatura en Historia presencial de la FFyL - UACH, en relación a la percepción que ellos tienen de su permanencia. Elaboración propia.

Fecha:

Facultad:

Sexo: F M I Pdad:

¿Tienes algún titulo profesional?: Sí Nou ¿Cuál (es)?

Irabaja: Sip Nop ¿En dónde trabaja?:

¿Qué razones te motivación para ingresar como alumno a la Licenciatura en Historia en modalidad presencial?

¿Cuánto interés tienes por estudiar esta carrera?

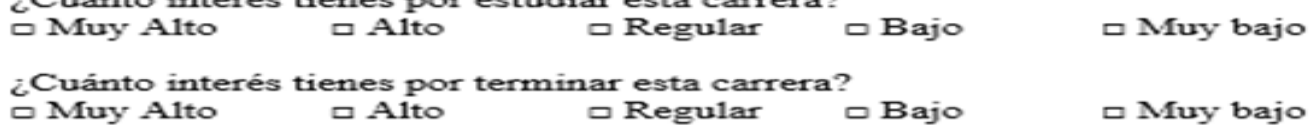

¿Cuải es el nivel de influencia que tendrán tus compañeros de clase sobre tu continuidad en esta carrera?

o Muy Alto a Alto a Regular b Bajo Muybajo

¿Cuál es el nivel de influencia que tendrán tus profesores sobre tu continuidad en esta carrera?

D Muy Alto a Alto a Regular a Bajo Muybajo

¿Cuál es el nivel de influencia que tendrá tu familia sobre tu continuidad en esta carrera? Muy Alto a Alto a Regular b Bajo Duy bajo

¿Cuál es el nivel de influencia que tendrán tus amigos sobre tu continuidad en esta carrera?

D Muy Alto a Alto a Regular a Bajo Duybajo

¿Cuál es el nivel de influencia que tendrán las posibilidades de un empleo en torno a la Licenciatura sobre tu continuidad en esta carrera?

b Muy Alto a Alto a Regular a Bajo Mubajo

¿Cuảl es el nivel de influencia que tendrá el obtener una beca o estimulo académico sobre tu continuidad en esta carrera?

o Muy Alto a Regular a Bajo alto Muybajo

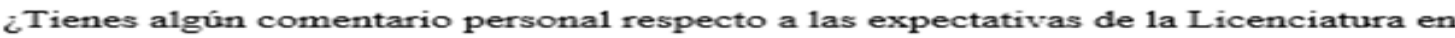
Historia?

¡MUCHAS GRACIAS POR SU RESPUESTAI 